\title{
Quantum-Mechanically Induced Asymmetry in the Phase Diagrams of Spin-Glass Systems
}

\author{
C. Nadir Kaplan ${ }^{1}$ and A. Nihat Berker ${ }^{1,2,3}$ \\ ${ }^{1}$ Department of Physics, Koç University, Sariyer 34450, Istanbul, Turkey \\ ${ }^{2}$ Feza Gürsey Research Institute, TÜBITAK - Bosphorus University, Çengelköy 81220, Istanbul, Turkey \\ ${ }^{3}$ Department of Physics, Massachusetts Institute of Technology, Cambridge, Massachusetts 02139, USA \\ (Received 22 September 2007; revised manuscript received 27 October 2007; published 16 January 2008) \\ The spin-1/2 quantum Heisenberg spin-glass system is studied in all spatial dimensions $d$ by \\ renormalization-group theory. Strongly asymmetric phase diagrams in temperature and antiferromagnetic \\ bond probability $p$ are obtained in dimensions $d \geq 3$. The asymmetry at high temperatures approaching \\ the pure ferromagnetic and antiferromagnetic systems disappears as $d$ is increased. However, the \\ asymmetry at low but finite temperatures remains in all dimensions, with the antiferromagnetic phase \\ receding from the ferromagnetic phase. A finite-temperature second-order phase boundary directly \\ between the ferromagnetic and antiferromagnetic phases occurs in $d \geq 6$, resulting in a new multicritical \\ point. In $d=3,4,5$, a paramagnetic phase reaching zero temperature intervenes asymmetrically between \\ the ferromagnetic and reentrant antiferromagnetic phases. There is no spin-glass phase in any dimension.
}

DOI: 10.1103/PhysRevLett.100.027204

A conspicuous finite-temperature effect of quantum mechanics is the critical temperature differentiation between ferromagnetic and antiferromagnetic systems [1-4]. This is a contrast to classical systems where, e.g., on loosepacked lattices ferromagnetic and antiferromagnetic systems are mapped onto each other and therefore have the same critical temperature. We find that this quantum effect is compounded and even more robust in spin-glass systems, which incorporate the passage from ferromagnetism and antiferromagnetism via quenched disorder.

Thus, in the present work, the phase diagrams of the spin-1/2 quantum Heisenberg spin-glass systems are calculated in all dimensions $d \geq 3$. In the space of temperature $T$ and concentration $p$ of antiferromagnetic bonds, remarkably asymmetric phase diagrams are obtained, in very strong contrast to the corresponding classical systems. Whereas, in the limit of $d \rightarrow \infty$, the differentiation of the critical temperatures of the ferromagnetic and antiferromagnetic pure systems disappears, the $T p$ phase diagrams remain strongly asymmetric at low but finite temperatures, where quantum fluctuations remain dominant independent of dimensionality. A direct second-order phase boundary between ferromagnetic and antiferromagnetic phases, also not seen in isotropic classical systems, is found in $d>5$. In lower $d$, a paramagnetic phase intervenes between the ferromagnetic and antiferromagnetic systems. Our calculation is an approximation for hypercubic lattices and, simultaneously, a lesser approximation for hierarchical lattices [5-15].

The spin- $1 / 2$ quantum Heisenberg spin-glass systems have the Hamiltonian $-\beta \mathcal{H}=\sum\langle i j\rangle J_{i j} \mathbf{s}_{i} \cdot \mathbf{s}_{j} \equiv$ $\sum_{\langle i j\rangle}-\beta \mathcal{H}(i, j)$, where $\langle i j\rangle$ denotes a sum over pairs of nearest-neighbor sites. $J_{i j}$ is equal to the ferromagnetic value of $J>0$ with probability $1-p$ and to the antiferromagnetic value of $-J<0$ with probability $p$. We solve this model by extending the Suzuki-Takano rescaling $[3,4,16-24]$ to nonuniform systems and to length-rescaling
PACS numbers: 75.10.Nr, 05.10.Cc, 05.45.Df, 64.60.ae

factor $b=3$, necessary for the a priori equivalent treatment of ferromagnetism and antiferromagnetism, followed by the essentially exact treatment $[25,26]$ of the quenched randomness giving the nonuniformity. In one dimension,

$$
\begin{aligned}
\operatorname{Tr}_{(j, k)} e^{-\beta \mathcal{H}} & =\operatorname{Tr}_{(j, k)} e^{\sum_{i}^{4 n}\{-\beta \mathcal{H}(i, j)-\beta \mathcal{H}(j, k)-\beta \mathcal{H}(k, l)\}} \\
& \simeq \prod_{i}^{4 n} \operatorname{tr}_{(j, k)} e^{\{-\beta \mathcal{H}(i, j)-\beta \mathcal{H}(j, k)-\beta \mathcal{H}(k, l)\}} \\
& =\prod_{i}^{4 n} e^{-\beta^{\prime} \mathcal{H}^{\prime}(i, l)} \simeq e^{\sum_{i}^{4 n}\left\{-\beta^{\prime} \mathcal{H}^{\prime}(i, l)\right\}} \\
& =e^{-\beta^{\prime} \mathcal{H}^{\prime}}
\end{aligned}
$$

where the sums and products $i$ are over every fourth spin along the chain, the traces are over all other spins, and $-\beta^{\prime} \mathcal{H}^{\prime}$ is the renormalized Hamiltonian. Thus, the commutation rules are correctly accounted for within four-site segments, at all successive length scales in the iterations of the renormalization-group transformation. The trace "tr" is performed by quantum algebra, as given below.

The rescaling is extended to dimensions $d>1$ by bondmoving, namely, by adding $b^{d-1}$ interactions resulting from the decimation of Eq. (1), to obtain the renormalized interaction strength $J_{i^{\prime} j^{\prime}}^{\prime}=R\left(\left\{J_{i j}\right\}\right)$, where $\left\{J_{i j}\right\}$ includes $b^{d}$ interactions of the unrenormalized system. The interaction constant values $\left\{J_{i j}\right\}$ are distributed with a quenched probability distribution $\mathcal{P}\left(J_{i j}\right)$ [25,26], which starts out as a double-delta function but quickly becomes complicated under its renormalization-group transformation, given by the convolution $\mathcal{P}^{\prime}\left(J_{i^{\prime} j^{\prime}}^{\prime}\right)=\int\left[\prod_{i j}^{i^{\prime} j^{\prime}} d J_{i j} \mathcal{P}\left(J_{i j}\right)\right] \delta\left[J_{i^{\prime} j^{\prime}}^{\prime}-\right.$ $\left.R\left(\left\{J_{i j}\right\}\right)\right]$. This equation actually involves $b^{d}$ convolutions (for example, 729 convolutions for the $d=6$ system discussed below), which are constituted of triplet convolutions of interactions in series (decimation) and pairwise convolutions of interactions in parallel (bond-moving). 
The quenched probability distribution $\mathcal{P}\left(J_{i j}\right)$ is kept numerically in terms of histograms. The number of histograms multiplicatively increases under rescaling, until a calculationally acceptable maximum is reached. After this point, the number of histograms is kept constant by implementing a binning procedure before each pairwise or triplet convolution. We employ a new binning procedure, in which bins are demarked so as to contain equal probabilities, as opposed to equal interaction intervals as done previously. Starting from the lowest $J$ value and moving to greater ones, histograms in each consecutive bin are combined, to interaction value $J=\Sigma p_{i} J_{i} / \Sigma p_{i}$ and imposed equal probability $p=\Sigma p_{i}=1 / n_{\text {bin }}$. In this process, histograms at the boundaries of bins are apportioned between the consecutive bins. Thus, our calculation has 125000 histograms after each decimation and 40000 histograms after each pairwise bond moving. The global flows of the quenched probability distributions yield the phase diagrams. Analysis of the unstable fixed points and unstable fixed distributions attracting the phase boundaries yields the order of the phase transitions.

Calculations are done for the quantum Heisenberg spinglass systems in integer dimensions. No finite-temperature phase transition occurs in $d=1,2$. The phase diagrams for $d=3,4,5,6,8,10$ are shown in Fig. 1. They are all strikingly asymmetric, especially in the middle $p$ and lowtemperature (would-be spin-glass phase) region. In $d=3$, our calculated ratio of the critical temperatures of the pure antiferromagnetic and ferromagnetic systems is $T_{C}^{\mathrm{AF}} / T_{C}^{F}=$ 1.48. This value is to be compared with the values of 1.13 found in the cubic lattice $[1,2]$ and 1.22 found in the $b=2$, $d=3$ hierarchical lattice [3,4]. This critical temperature difference is consistent with the lower ground-state energy of the antiferromagnetic system, as calculated [27] in $d=$ 3 . Our calculated ratios of the antiferromagnetic and ferromagnetic critical temperatures, for $d=4,5,6,8,10$ decrease as $1.22,1.12,1.07,1.02,1.01$, respectively. On the other hand, it is seen that although the phase boundaries leading to the pure ferromagnetic and antiferromagnetic critical points regain symmetry as $d$ is increased, the lowtemperature phase diagrams remain asymmetric. The ferromagnetic phase penetrates the antiferromagnetic region at low temperatures. Thus, quantum fluctuations present at low temperatures favor the ferromagnetic phase over the antiferromagnetic phase. In $d \geq 6$, a second-order phase boundary occurs directly between the ferromagnetic and antiferromagnetic phases, as is not seen in isotropic classical spin-glass systems. A new multicritical point occurs where all 3 second-order boundaries meet.

The phase transition, between ordered phases, that is driven by quenched randomness presents a contrast to phase transitions between ordered phases driven by a systemwise uniform interaction. The latter phase transition is obtained, at low temperatures, by driving a uniform interaction that favors another ordered phase over the existing one. Under these conditions, essentially the entire system remains in one ordered phase until the phase transition point is reached, when essentially the entire system changes over to the other phase. Throughout this process, the ordered domains are compact and have fractal dimen-
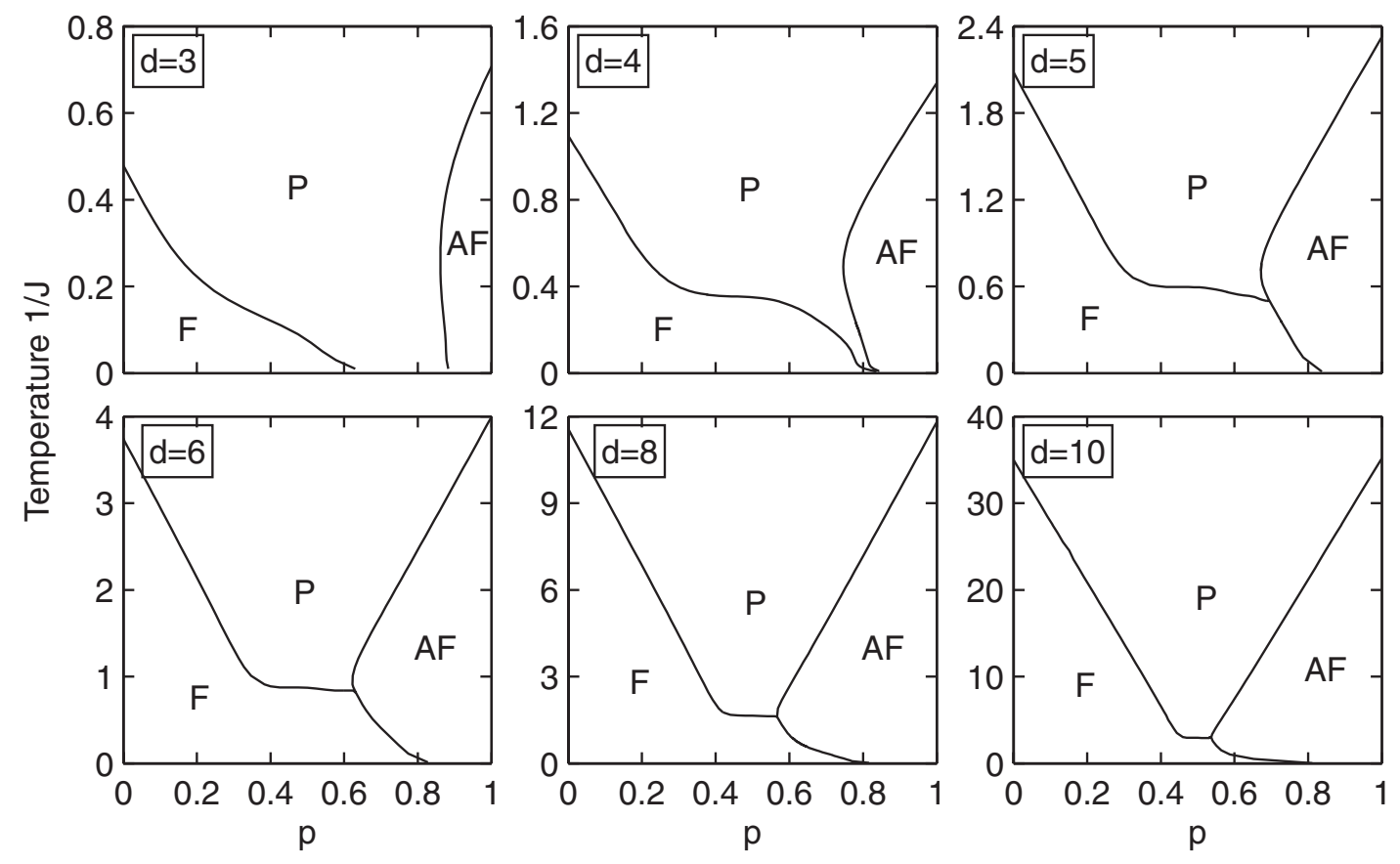

FIG. 1. Phase diagrams of the quantum Heisenberg spin-glass systems in temperature $1 / J$ versus antiferromagnetic bond concentration $p$ for $d=3$ to 10 . All transitions are second-order, between the ferromagnetic (F), antiferromagnetic (AF), and paramagnetic $(\mathrm{P})$ phases. 
TABLE I. The two-site basis states, with the corresponding parity $(p)$, total spin $(s)$, and total spin $z$ component $\left(m_{s}\right)$ quantum numbers. The state $\left|\phi_{3}\right\rangle$ is obtained by spin reversal from $\left|\phi_{1}\right\rangle$. The renormalized two-site Hamiltonian $-\beta^{\prime} H^{\prime}(i, l)$ is diagonal in this set, with the diagonal elements of $\left|\phi_{1-3}\right\rangle$ and $\left|\phi_{4}\right\rangle$ being $\frac{1}{4} J^{\prime}+G^{\prime}$ and $-\frac{3}{4} J^{\prime}+G^{\prime}$, respectively.

\begin{tabular}{llcl}
\hline \hline$p$ & $s$ & $m_{s}$ & Two-Site Eigenstates \\
\hline+ & 1 & 1 & $\left|\phi_{1}\right\rangle=|\uparrow \uparrow\rangle$ \\
+ & 1 & 0 & $\left|\phi_{2}\right\rangle=\frac{1}{\sqrt{2}}\{|\uparrow \downarrow\rangle+|\downarrow \uparrow\rangle\}$ \\
- & 0 & 0 & $\left|\phi_{4}\right\rangle=\frac{1}{\sqrt{2}}\{|\uparrow \downarrow\rangle-|\downarrow \uparrow\rangle\}$ \\
\hline \hline
\end{tabular}

sionality equal to spatial dimensionality, which translates to having a renormalization-group eigenvalue exponent of $y=d$, the condition for a first-order transition [28]. By contrast, the phase transition with quenched randomness is obtained, at low temperatures, by driving quenched local interactions that favor the other ordered phase over the existing phase. This means, for example, increasing the number of random antiferromagnetic bonds when the system is in the ferromagnetic phase. Under these conditions, the ferromagnetic domains avoid the random localities of antiferromagnetic bonds in the system. The ferromagnetically ordered domains loose weight as the transition is approached, so that the average magnetization decreases. At the phase transition, the ordered domains are not compact and have fractal dimensionality less than the spatial dimensionality, so that the magnetization is zero. This translates to the renormalization-group eigenvalue exponent $y<d$, meaning a second-order phase transition. The converse happens when the phase transition is approached from the opposite side, with noncompact antiferromagnetic domains avoiding the random localities of the ferromagnetic bonds. Similarly, in another recently studied system with quenched randomness, second-order transitions between ferromagnetic and layered ordered phases and between antiferromagnetic and columnar ordered phases, are obtained in the exact solution of classical anisotropic spinglasses on a hierarchical lattice [29]. Thus, we find that whereas phase transitions between ordered phases are first order when driven by a uniform interaction, they are second order when driven by quenched randomness.

In $d=3,4,5$, the paramagnetic phase reaching zero temperature (as an extremely narrow sliver in $d=5$ ) intervenes between the ferromagnetic and antiferromagnetic phases. In all cases, the ferromagnetic phase penetrates, reaching the high $p$ values of 0.63 and 0.83 , respectively, in $d=3$, where there is a zero-temperature paramagnetic interval, and $d \geq 4$, where there is no zero-temperature paramagnetic interval. The antiferromagnetic phase recedes at low temperatures, thereby showing a reentrant phase boundary [26].

There is no spin-glass phase, in the quantum system, in any dimension. The quantum version of the SherringtonKirkpatrick model [30], namely, the spin-1/2 quantum Heisenberg model with equivalent-neighbor interactions, with a symmetric Gaussian distribution, studied from the high-temperature side, yields a finite-temperature phase transition, which has been interpreted as a transition to a low-temperature spin-glass phase [31]. This model should be similar to our studied models at $p=0.5$ in the large $d$ limit. Thus, we also find a finite-temperature phase transition (Fig. 1), but the low-temperature phase is explicitly a ferromagnetic phase with quenched bond randomness. The latter phase has considerable amount of short-range antiferromagnetic correlations, as seen in Ref. [32].

We are grateful to $\mathrm{M}$. Hinczewski and $\mathrm{H}$. Nishimori for useful comments. This research was supported by the Scientific and Technological Research Council (TÜBITAK) and by the Academy of Sciences of Turkey. Appendix. - The operators $-\beta^{\prime} \mathcal{H}^{\prime}(i, l)$ and $-\beta \mathcal{H}(i, j)-\beta \mathcal{H}(j, k)-\beta \mathcal{H}(k, l)$ of Eq. (1) act on two-site and four-site states, respectively, where at each site the spin is in quantum state $\sigma=\uparrow$ or $\downarrow$. The trace in Eq. (1) is, in terms of matrix elements [3],

$$
\left\langle u_{i} z_{l}\left|e^{-\beta^{\prime} \mathcal{H}^{\prime}(i, l)}\right| \bar{u}_{i} \bar{z}_{l}\right\rangle=\sum_{v_{j}, w_{k}}\left\langle u_{i} v_{j} w_{k} z_{l}\left|e^{-\beta \mathcal{H}(i, j)-\beta \mathcal{H}(j, k)-\beta \mathcal{H}(k, l)}\right| \bar{u}_{i} v_{j} w_{k} \bar{z}_{l}\right\rangle,
$$

TABLE II. The four-site basis states, with the corresponding parity $(p)$, total spin $(s)$, and total spin $z$ component $\left(m_{s}\right)$ quantum numbers. The states $\left|\psi_{4,5}\right\rangle,\left|\psi_{8}\right\rangle,\left|\psi_{13,14}\right\rangle$ are obtained by spin reversal from $\left|\psi_{2,1}\right\rangle,\left|\psi_{6}\right\rangle,\left|\psi_{9,10}\right\rangle$, respectively.

\begin{tabular}{|c|c|c|c|}
\hline$p$ & $s$ & $m_{s}$ & Four-Site Eigenstates \\
\hline+ & 2 & 2 & $\left|\psi_{1}\right\rangle=|\uparrow \uparrow \uparrow \uparrow\rangle$ \\
\hline+ & 2 & 1 & $\left.\left|\psi_{2}\right\rangle=\frac{1}{2}\{|\uparrow \uparrow \uparrow \downarrow\rangle+|\uparrow \uparrow \downarrow \uparrow\rangle+|\uparrow \downarrow \uparrow \uparrow\rangle+\mid \downarrow \uparrow \uparrow \uparrow\}\right\}$ \\
\hline+ & 2 & 0 & $\left.\left|\psi_{3}\right\rangle=\frac{1}{\sqrt{6}}\{|\uparrow \uparrow \downarrow \downarrow\rangle+|\uparrow| \uparrow \downarrow\rangle+|\uparrow \downarrow \downarrow \uparrow\rangle+|\downarrow \uparrow \uparrow \downarrow\rangle+|\downarrow \uparrow \downarrow \uparrow\rangle+|\downarrow \downarrow \uparrow\rangle\right\}$ \\
\hline+ & 1 & 1 & $\left|\psi_{6}\right\rangle=\frac{1}{2}\{|\uparrow \uparrow \uparrow \downarrow\rangle-|\uparrow \uparrow \downarrow \uparrow\rangle-|\uparrow \downarrow \uparrow \uparrow\rangle+|\downarrow \uparrow \uparrow \uparrow\rangle\}$ \\
\hline+ & 1 & 0 & $\left|\psi_{7}\right\rangle=\frac{1}{\sqrt{2}}\{|\downarrow \uparrow \uparrow \downarrow\rangle-|\uparrow \downarrow \downarrow \uparrow\rangle\}$ \\
\hline- & 1 & 1 & $\left|\psi_{9}\right\rangle=\frac{1}{2}\{|\uparrow \uparrow \uparrow\rangle-|\uparrow \uparrow \downarrow \uparrow\rangle+|\uparrow \downarrow \uparrow \uparrow\rangle-|\downarrow \uparrow \uparrow\rangle\},\left|\psi_{10}\right\rangle=\frac{1}{2}\{|\uparrow \uparrow \uparrow \downarrow\rangle+|\uparrow \uparrow \downarrow \uparrow\rangle-|\uparrow \downarrow \uparrow \uparrow\rangle-\mid \downarrow \uparrow \uparrow \uparrow\}$ \\
\hline- & 1 & 0 & $\left|\psi_{11}\right\rangle=\frac{1}{\sqrt{2}}\{|\uparrow \downarrow \uparrow \downarrow\rangle-|\downarrow \uparrow \uparrow \uparrow\rangle\},\left|\psi_{12}\right\rangle=\frac{1}{\sqrt{2}}\{|\uparrow \uparrow \downarrow \downarrow\rangle-|\downarrow \downarrow \uparrow\rangle\}$ \\
\hline+ & 0 & 0 & $\left.\left|\psi_{15}\right\rangle=\frac{1}{2}\{|\uparrow \uparrow \downarrow\rangle-|\uparrow \downarrow \uparrow \downarrow\rangle-|\downarrow \uparrow \downarrow \uparrow\rangle+|\downarrow \downarrow \uparrow \uparrow\rangle\},\left|\psi_{16}\right\rangle=\frac{1}{\sqrt{12}}\{|\uparrow \uparrow \downarrow\rangle+|\uparrow| \uparrow \downarrow\rangle-2|\uparrow \downarrow \uparrow\rangle-2|\downarrow \uparrow \uparrow \downarrow\rangle+|\downarrow \uparrow \downarrow \uparrow\rangle+|\downarrow \downarrow \uparrow \uparrow\rangle\right\}$ \\
\hline
\end{tabular}


TABLE III. Diagonal matrix blocks of the unrenormalized three-site Hamiltonian $-\beta H(i, j)-\beta H(j, k)-\beta H(k, l)$. The Hamiltonian being invariant under spin reversal, the spin-flipped matrix elements are not shown. The additive constant $3 G$ at the diagonal elements is not shown. The interaction constants $J_{1}, J_{2}$, $J_{3}$ which are in general unequal due to quenched randomness, are from $-\beta \mathcal{H}(i, j),-\beta \mathcal{H}(j, k),-\beta \mathcal{H}(k, l)$, respectively. The block between $\psi_{7,11,12}$ is the same as the block between $\psi_{6,9,10}$.

\begin{tabular}{cccc}
\hline \hline & $\psi_{1}$ & $\psi_{2}$ & $\psi_{3}$ \\
\hline$\psi_{1}$ & $\frac{1}{4}\left(J_{1}+J_{2}+J_{3}\right)$ & 0 & 0 \\
$\psi_{2}$ & 0 & $\frac{1}{4}\left(J_{1}+J_{2}+J_{3}\right)$ & 0 \\
$\psi_{3}$ & 0 & 0 & $\frac{1}{4}\left(J_{1}+J_{2}+J_{3}\right)$ \\
\hline \hline
\end{tabular}

\begin{tabular}{lccc}
\hline \hline & $\psi_{6}$ & $\psi_{9}$ & $\psi_{10}$ \\
\hline$\psi_{6}$ & $\frac{1}{4}\left(-J_{1}+J_{2}-J_{3}\right)$ & $\frac{1}{2}\left(J_{1}-J_{3}\right)$ & 0 \\
$\psi_{9}$ & $\frac{1}{2}\left(J_{1}-J_{3}\right)$ & $-\frac{1}{4}\left(J_{1}+J_{2}+J_{3}\right)$ & $\frac{1}{2} J_{2}$ \\
$\psi_{10}$ & 0 & $\frac{1}{2} J_{2}$ & $\frac{1}{4}\left(J_{1}-J_{2}+J_{3}\right)$ \\
\hline \hline
\end{tabular}

\begin{tabular}{ccc}
\hline & $\psi_{15}$ & $\psi_{16}$ \\
\hline$\psi_{15}$ & $-\frac{3}{4} J_{2}$ & $\frac{\sqrt{3}}{4}\left(J_{1}+J_{3}\right)$ \\
$\psi_{16}$ & $\frac{\sqrt{3}}{4}\left(J_{1}+J_{3}\right)$ & $\frac{1}{4}\left(-2 J_{1}+J_{2}-2 J_{3}\right)$ \\
\hline \hline
\end{tabular}

where $u_{i}, v_{j}, w_{k}, z_{l}, \bar{u}_{i}, \bar{z}_{l}$ are single-site state variables. Thus, Eq. (a1) is the contraction of a $16 \times 16$ matrix into a $4 \times 4$ matrix. Basis states that are simultaneous eigenstates of parity $(p)$, total spin magnitude $(s)$, and total spin $z$ component $\left(m_{s}\right)$ block-diagonalize these matrices and thereby make Eq. (a1) manageable. These sets of 4 twosite and 16 four-site eigenstates, denoted by $\left\{\left|\phi_{p}\right\rangle\right\}$ and $\left\{\left|\psi_{q}\right\rangle\right\}$ respectively, are given in Tables I and II. The diagonal blocks are given in Tables I and III. Because of the microscopic randomness of the spin-glass problem, the four-site Hamiltonian mixes states of different parity, as seen in Table III. Equation (a1) is thus rewritten as

$$
\begin{aligned}
& \left\langle\phi_{p}\left|e^{-\beta^{\prime} \mathcal{H}^{\prime}(i, l)}\right| \phi_{\bar{p}}\right\rangle=\sum_{\substack{u, z, u_{i} \\
\bar{z}, v, w}} \sum_{q, \bar{q}}\left\langle\phi_{p} \mid u_{i} z_{l}\right\rangle\left\langle u_{i} v_{j} w_{k} z_{l} \mid \psi_{q}\right\rangle \\
& \times\left\langle\psi_{q}\left|e^{-\beta \mathcal{H}(i j)-\beta \mathcal{H}(j, k)-\beta \mathcal{H}(k, l)}\right| \psi_{\bar{q}}\right\rangle \\
& \times\left\langle\psi_{\bar{q}} \mid \bar{u}_{i} v_{j} w_{k} \bar{z}_{l}\right\rangle\left\langle\bar{u}_{i} \bar{z}_{l} \mid \phi_{\bar{p}}\right\rangle .
\end{aligned}
$$

There are only two rotation-symmetry independent elements of $\left\langle\phi_{p}\left|e^{-\beta^{\prime} \mathcal{H}^{\prime}(i, l)}\right| \phi_{\bar{p}}\right\rangle \equiv\left\langle\phi_{p} \| \phi_{\bar{p}}\right\rangle$ in Eq. (a2), which have $p=\bar{p}=1,4$ (thereby leading to one renormalized interaction constant $J^{\prime}$ and the additive constant $\left.G^{\prime}\right)$. From Eq. (a2), $\left\langle\phi_{1} \| \phi_{1}\right\rangle=\left\langle\psi_{1} \| \psi_{1}\right\rangle+$ $\frac{1}{2}\left\langle\psi_{2} \| \psi_{2}\right\rangle+\frac{1}{6}\left\langle\psi_{3} \| \psi_{3}\right\rangle+\frac{1}{2}\left\langle\psi_{6} \| \psi_{6}\right\rangle+\frac{1}{2}\left\langle\psi_{7} \| \psi_{7}\right\rangle+\frac{1}{2} \times$ $\left\langle\psi_{9} \| \psi_{9}\right\rangle-\left\langle\psi_{9} \| \psi_{10}\right\rangle+\frac{1}{2}\left\langle\psi_{10} \| \psi_{10}\right\rangle+\frac{1}{3}\left\langle\psi_{16} \| \psi_{16}\right\rangle$ and $\left\langle\phi_{4} \| \phi_{4}\right\rangle=\left\langle\psi_{9} \| \psi_{9}\right\rangle+2\left\langle\psi_{9} \| \psi_{10}\right\rangle+\left\langle\psi_{10} \| \psi_{10}\right\rangle+\frac{1}{2} \times$ $\left\langle\psi_{11} \| \psi_{11}\right\rangle+\left\langle\psi_{11} \| \psi_{12}\right\rangle+\frac{1}{2}\left\langle\psi_{12} \| \psi_{12}\right\rangle+\left\langle\psi_{15} \| \psi_{15}\right\rangle$, with $\left\langle\psi_{q} \| \psi_{\bar{q}}\right\rangle \equiv\left\langle\psi_{q}\left|e^{-\beta \mathcal{H}(i, j)-\beta \mathcal{H}(j, k)-\beta \mathcal{H}(k, l)}\right| \psi_{\bar{q}}\right\rangle . \quad$ From Table I, the renormalized interaction constant is given by $J^{\prime}=\ln \left(\left\langle\phi_{1} \| \phi_{1}\right\rangle /\left\langle\phi_{4} \| \phi_{4}\right\rangle\right)$.
[1] G. S. Rushbrooke and P. J. Wood, Mol. Phys. 6, 409 (1963).

[2] J. Oitmaa and W. Zheng, J. Phys. Condens. Matter 16, 8653 (2004).

[3] A. Falicov and A. N. Berker, Phys. Rev. B 51, 12458 (1995).

[4] M. Hinczewski and A. N. Berker, Eur. Phys. J. B 48, 1 (2005).

[5] A. N. Berker and S. Ostlund, J. Phys. C 12, 4961 (1979).

[6] R. B. Griffiths and M. Kaufman, Phys. Rev. B 26, 5022 (1982).

[7] M. Kaufman and R. B. Griffiths, Phys. Rev. B 30, 244 (1984).

[8] A. Erbaş, A. Tuncer, B. Yücesoy, and A. N. Berker, Phys. Rev. E 72, 026129 (2005).

[9] M. Hinczewski and A. N. Berker, Phys. Rev. E 73, 066126 (2006).

[10] M. Hinczewski, Phys. Rev. E 75, 061104 (2007).

[11] Z. Zhang, L. Rong, and S. Zhou, Physica (Amsterdam) 377A, 329 (2007).

[12] Z. Zhang, S. Zhou, and T. Zou, Eur. Phys. J. B 56, 259 (2007).

[13] H. D. Rozenfeld and D. ben-Avraham, Phys. Rev. E 75, 061102 (2007).

[14] H. D. Rozenfeld, S. Havlin, and D. ben-Avraham, New J. Phys. 9, 175 (2007).

[15] E. Khajeh, S. N. Dorogovtsev, and J.F. F. Mendes, Phys. Rev. E 75, 041112 (2007).

[16] M. Suzuki and H. Takano, Phys. Lett. A 69, 426 (1979).

[17] H. Takano and M. Suzuki, J. Stat. Phys. 26, 635 (1981).

[18] P. Tomczak, Phys. Rev. B 53, R500 (1996).

[19] P. Tomczak and J. Richter, Phys. Rev. B 54, 9004 (1996).

[20] P. Tomczak and J. Richter, J. Phys. A 36, 5399 (2003).

[21] M. Hinczewski and A. N. Berker, Eur. Phys. J. B 51, 461 (2006).

[22] M. Hinczewski and A. N. Berker, arXiv:cond-mat/ 0607171.

[23] C. N. Kaplan, A. N. Berker, and M. Hinczewski (to be published).

[24] O.S. Saryyer, A. N. Berker, and M. Hinczewski, arXiv:0704.1064.

[25] A. Falicov, A. N. Berker, and S. R. McKay, Phys. Rev. B 51, 8266 (1995).

[26] G. Migliorini and A. N. Berker, Phys. Rev. B 57, 426 (1998).

[27] H. Nishimori and S. J. Miyake, Prog. Theor. Phys. 73, 18 (1985).

[28] S. Ostlund and A. N. Berker, Phys. Rev. B 21, 5410 (1980).

[29] C. Güven, A. N. Berker, M. Hinczewski, and H. Nishimori (to be published).

[30] D. Sherrington and S. Kirkpatrick, Phys. Rev. Lett. 35, 1792 (1975).

[31] A. J. Bray and M.A. Moore, J. Phys. C 13, L655 (1980).

[32] B. Yücesoy and A. N. Berker, Phys. Rev. B 76, 014417 (2007). 